Method Computerised article search conducted using the electronic databases Medline, Science Direct and the Cochrane Library for the literature review. Interviews were conducted with the child's mother and community paediatrician.

Results After sleep hygiene optimisation and behavioural interventions, sleep onset latency had decreased by one hour. After melatonin, sleep-onset latency; frequency of night terrors and other nocturnal awakenings and daytime behaviour had improved. Total sleep time had increased by $4 \mathrm{~h} 30 \mathrm{~min}$.

Conclusion Further research should be done to set up more sleep clinics nationwide. Official guidelines or practice pathways should be made to guide professionals in how to manage sleep problems in ASD.

\section{G203(P) STATUS DYSTONICUS PRESENTING IN AN ACUTE SETTING IN ASSOCIATION WITH VIRAL ILLNESSES}

SA Jaleel, J Balfe, E Curtis, D McDonald. Department of Developmental Paediatrics, The Adelaide and Meath Hospital Incorporating the National Children's Hospital, Dublin, Ireland

\subsection{6/archdischild-2015-308599.197}

Aims We report 2 cases of status dystonicus seen in our hospital in 2014. Status dystonicus is a rare condition with a potentially life-threatening outcome. The patients' background, clinical features, management, likely triggers, differential diagnosis and outcome is discussed.

Methods Review of charts.

- Review of existing literature.

Description Case1:A 9-year-old female with dystonic quadriparesis GMFCS class IV, intellectual disability and gastrostomy feeds was admitted with a febrile illness (pneumonia) and treated with intravenous antibiotics, oxygen and fluid support. Her dystonic movements had not required treatment before this.

During the admission, she developed severe abnormal movements and unusual posturing with sustained hyperpyrexia, sweating and rising creatinine kinase $(\mathrm{CK})>23,000$. Blood and urine cultures were negative. She was intubated and ventilated, transferred to PICU, and received chloral hydrate, clonidine, and oseltamivir. She was subsequently confirmed as having Influenza A, H3N2 strain. During recovery, sedation was gradually weaned; however, dystonic movements recurred, requiring institution of a slower weaning regimen. She went home on trihexyphenidyl and remains well.

Case 2: A 5-year-old male with microcephaly, spastic quadriparesis GMFCS class V (intra-thecal baclofen pump), visual impairment, profound intellectual disability, recurrent urinary tract infections (UTI), nephcalcinosis, gastrostomy feeds was admitted with Pseudomonas UTI and treated with ciprofloxacin.

His recovery was complicated by norovirus gastro-enteritis with dehydration, pre-renal failure and increasingly severe dystonic posturing (tongue protrusion, sustained muscle contractions, ophisthotonus) with fever, sweating and rising CK (> 23000). He was treated with: transfer to HDU, close management of fluid and electrolyte balance, sedation with chloral hydrate, clonidine and midazolam.

Resolution of the movement disorder and fever and normalisation of CK followed. He re-presented a month later with similar symptomatology; however, early treatment with hydration, clonidine and chloral hydrate appeared to halt progression to status dystonicus. He went home on low-dose clonidine and remains well.
Conclusion Status dystonicus is a rare condition with a high morbidity and mortality. A rising CK, severe dystonic movements and metabolic derangements suggest the diagnosis. Maintaining a high index of suspicion can identify such cases early and halt further progression. $\mathrm{CK}$ is simple test to monitor response to treatment.

\section{G204(P) OSTEOSARCOMA CELL CULTURE ON COLLAGEN SURFACES AND IN HYPOXIA ALTERS MMP EXPRESSION}

S Nisar, M Birch, K Rankin. Musculoskeletal Research Group, Newcastle University, Newcastle-Upon-Tyne, UK

\subsection{6/archdischild-2015-308599.198}

Osteosarcoma is the most common primary malignant bone tumour in children. The survival rate has not improved much over the last 25 years, and therefore there is a lot to learn about the pathogenesis of this cancer. The interactions of tumour cells with their environment and hypoxia have been identified as key drivers of tumour growth and metastasis. Matrix-metallo proteinases (MMPs) are involved in this process. MMPs are zinc-endopeptidases that are able to degrade the extra-cellular matrix and are over-expressed in many tumours. Membrane-type (MT1)MMP and MMP-2 expression is positively associated with tumour progression in a range of tumours, but their role is not well characterised in osteosarcoma.

Two osteosarcoma cell lines were cultured on culture plastic or collagen surfaces in either normoxia or hypoxia. Proliferation was assessed using the SRB assay which showed osteosarcoma cells proliferate slightly slower in hypoxia. Immunofluorescence microscopy was employed to visualise MT1-MMP - this revealed MT1-MMP packaging and localisation was altered in hypoxia and there was formation of invadopodia on collagen. Gelatinase expression, assessed using zymography of supernatants, demonstrated increased proMMP-2 activation by cells cultured on collagen, particularly by U2OS cells. Cell lysates were probed for MT1-MMP using western blotting. ELISA of the culture supernatant was used to measure TIMP-2 expression. Less active MT1-MMP was detected in the lysates of the U2OS cells which coincided with a decreased amount of TIMP-2 detected in the supernatant.

This study contributes to our understanding of the activation of MMPs and the possible role of MT1-MMP in this regard.

\section{G205(P) FIBRODYSPLASIA OSSIFICANS PROGRESSIVA (FOP) AN UNFAMILIAR DISEASE THAT IS NOW IMPORTANT TO DIAGNOSE}

${ }^{1} \mathrm{R}$ Keen, ${ }^{2} \mathrm{~B}$ Jacobs. 'Metabolic Bone Unit, Royal National Orthopaedic Hospital, Stanmore; ${ }^{2}$ Paediatrics, Royal National Orthopaedic Hospital, Stanmore

\subsection{6/archdischild-2015-308599.199}

Background FOP is a rare but disabling condition characterised by congenital malformation of the great toes and progressive heterotopic endochondral ossification (HEO). FOP is the most catastrophic disorder of HEO in humans.

Flare-ups are episodic; immobility is cumulative. The discovery of the ACVR1 gene as the cause of FOP has allowed identification of possible therapeutic targets. Palovarotene, a retinoic acid receptor gamma agonist, is currently in Phase 2 clinical trials to reduce $\mathrm{HEO}$ during acute flares. 